\title{
ON THE FIXED POINTS OF MÖBIUS TRANSFORMATIONS IN $R^{n}$
}

\section{LARS V. AHLFORS*}

It has been known for a long time that Möbius transformations in $\boldsymbol{R}^{n}$ can be expressed in the form

$$
g x=(a x+b)(c x+d)^{-1}
$$

where the coefficients $a, b, c, d$ are Clifford numbers subject to certain restrictions, but very little use has been made of this observation. The writer has become convinced that this approach to Möbius transformations has very important advantages. It is not only a natural generalization of the use of two by two matrices in the classical case, but the formula (1) also yields an automatic extension from $n$ to $n+1$ dimensions. In this paper, which is still a preliminary report, we shall address the problem of finding and classifying the fixed point structure of $g$ in terms of the coefficients.

To my knowledge the first use of Clifford numbers in this connection was by K. Th. Vahlen [Va] who, in 1902, derived necessary and sufficient conditions for (1) to represent a Möbius transformation. His paper was forgotten until revived by H. Maass [Ma] in 1949. For more recent applications of Clifford algebras to Möbius groups see [Lo-La], [Fi] and [Ah 1, 2].

The relevant facts about Clifford numbers are reviewed in Section 1, mostly without proofs. Details can be found for instance in [Po]. Section 2 is a slightly different approach to Vahlen and Maass. The main part of the paper is in Sections 4-6 and deals with the identification of hyperbolic, elliptic, and parabolic transformations. This identification will be purely in terms of the coefficients $a, b, c, d$. In other words, when the coefficients are given the paper will provide the means to determine whether the transformation $g$ is or is not hyperbolic, elliptic, or parabolic. Section 7 illustrates this through an example worked out in some detail.

\footnotetext{
* Research supported by the National Science Foundation
} 


\section{Properties of the Clifford numbers}

The Clifford algebra $A_{n}$ shall be the associative algebra over the reals generated by elements $e_{1}, \ldots, e_{n}$ subject to the relations $e_{h}^{2}=-1$ and $e_{h} e_{k}=-e_{k} e_{h}, h \neq k$, and no others. Every $a \in A_{n}$ has a development of the form

$$
a=a_{0}+\sum a_{v} E_{v}
$$

where $a_{0}$ and $a_{v}$ are real and the sum ranges over all multi-indices $v=\left(v_{1}, \ldots, v_{p}\right)$ with $0<v_{1}<v_{2}<\ldots<v_{p} \leqq n$, and $E_{v}=e_{v_{1}} \ldots e_{v_{p}}$. The term $a_{0}$, referred to as the real part of $a$, is regarded as corresponding to the empty multi-index with $p=0$.

$A_{n}$ is a vector space of dimension $2^{n}$. It is a sum of the spaces $A_{n}^{p}$ spanned by the $E_{v}$ with fixed $p$. The sum of the $A_{n}^{p}$ with even $p$ will be denoted by $A_{n}^{0}$, while $A_{n}^{1}$ refers to odd $p . A_{n}^{(0)}$ is a subalgebra of $A_{n}$.

We shall identify $\boldsymbol{R}^{n}$ with the subspace spanned by $1, e_{1}, \ldots, e_{n-1}$. This is Vahlen's choice, similar to the identification of $\boldsymbol{R}^{2}$ with $C=A_{1}$. For other reasons it has become more common to identify $\boldsymbol{R}^{n}$ with $A_{n}^{1}$. Each choice has its advantages and disadvantages, but we prefer to follow Vahlen in spite of the unsymmetric role of 1 . We shall refer to the $x=x_{0}+x_{1} e_{1}+\ldots+x_{n-1} e_{n-1}$ as vectors to be regarded as elements of $A_{n-1}$ or $\boldsymbol{R}^{n}$ as the case may be.

The algebra $A_{n}$ has three important involutions, similar to complex conjugation. The first, or main involution, is the isomorphism $a \rightarrow a^{\prime}$ obtained by replacing each $e_{h}$ by $-e_{h}$, thereby replacing each $a \in A_{n}^{p}$ by $a^{\prime}=(-1)^{p} a$. It is obvious that $(a+b)^{\prime}=a^{\prime}+b^{\prime}$ and $(a b)^{\prime}=a^{\prime} b^{\prime}$.

The second involution, or reversion, is an anti-isomorphism $a \rightarrow a^{*}$ obtained by reversing the order of the factors $e_{v_{h}}$ in each $E_{v}$. It satisfies $(a b)^{*}=b^{*} a^{*}$, and $a \in A_{n}^{p}$ implies $a^{*}=(-1)^{p(p-1) / 2} a$. The third involution is a combination of the two others, and we shall write $\bar{a}=a^{* *}=a^{* \prime}$. It is again an anti-isomorphism, $(a b)^{-}=$ $\bar{b} \bar{a}$ and $\bar{a}=(-1)^{p(p+1) / 2} a$ if $a \in A_{n}^{p}$. The notations $a^{\prime}, a^{*}, \bar{a}$ are taken from Vahlen and Maass; they are not standardized.

For vectors, $x^{*}=x$ and $x^{\prime}=\bar{x}$; out of habit I prefer to use $\bar{x}$. Furthermore, $x \bar{x}=|x|^{2}$, the euclidean square norm. It follows that every non-zero vector is invertible with $x^{-1}=\bar{x} /|x|^{2}$. For two vectors,

$$
x \bar{y}+y \bar{x}=2(x, y)
$$

where $(x, y)$ is the inner product. The square norm extends to $A_{n}$ by writing

$$
|a|^{2}=a_{0}^{2}+\sum a_{v}^{2}
$$

when $a$ is given by (2). However, it is not universally true that $|a|^{2}=a \bar{a}=\bar{a} a$.

The Clifford group $\Gamma_{n}$ consists of all $a \in A_{n-1}$ which can be written as products of non-zero vectors in $\boldsymbol{R}^{n}$. It is obviously a subgroup of the multiplicative group of $A_{n-1}$. Elements of the Clifford group do satisfy $|a|^{2}=a \bar{a}$, from which it follows that $|a b|=|a||b|$. We draw attention to the seeming inconsistency in the choice of 
subscripts, which is due to a preference for working with $\boldsymbol{R}^{n}$ rather than $\boldsymbol{R}^{n-1}$. Again, our notation is not standard.

There is another characterization of $\Gamma_{n}$ which plays a central role and is often taken as the primary definition. It is seen by repeated use of (4) that if $a$ is in the Clifford group and $x$ is a vector, then $a x a^{\prime-1}$ is again a vector. The mapping $x \rightarrow a x a^{\prime-1}$ is obviously linear, and since $\left|a x a^{\prime-1}\right|=|x|$ it is a euclidean isometry. What is more, it is always sense-preserving. In other words, to every $a \in \Gamma_{n}$ there corresponds a matrix $\varrho(a) \in \mathrm{SO}(n)$ such that $a x a^{\prime-1}=\varrho(a) x$ for all $x \in \boldsymbol{R}^{n}$. Conversely, every matrix in $\mathrm{SO}(n)$ can be obtained in this way. The mapping $a \rightarrow \varrho(a)$ is a homomorphism with center $\boldsymbol{R} \backslash\{0\}$.

To connect with Vahlen I start from an algebraic definition which makes little sense by itself. The name Clifford matrix is historically unjustified, but I have chosen it to pay homage to an important thinker and originator, without thereby wishing to detract from Vahlen's just share.

Definition. The matrix $g=\left(\begin{array}{ll}a & b \\ c & d\end{array}\right)$ is a Clifford matrix in dimension $n$ if the following conditions are fulfilled:

(i) $a, b, c, d \in \Gamma_{n} \cup\{0\}$,

(ii) $a d^{*}-b c^{*}=1$,

(iii) $a c^{-1}$ and $c^{-1} d \in \boldsymbol{R}^{n}$ if $c \neq 0$,

(iii') $d b^{-1}$ and $b^{-1} a \in \boldsymbol{R}^{n}$ if $b \neq 0$.

Some preliminary comments are in order. Condition (ii) makes it impossible for two elements of $g$ in the same row or column to be simultaneously zero. The condition $a c^{-1} \in \boldsymbol{R}^{n}$ is equivalent to $c^{*} a=a^{*} c \in \boldsymbol{R}^{n}$. This follows from $\left(c^{* \prime}\right)^{-1}=$ $c /|c|^{2}$ and $c^{*} a=c^{*}\left(a c^{-1}\right) c$. Similarly, $c d^{*}, b^{*} d, a b^{*} \in \boldsymbol{R}^{n}$.

If $b$ and $c$ are both $\neq 0$, conditions (iii) and (iii') are equivalent. For instance, (ii) and (iii) imply $b=a d^{*} c^{*-1}-c^{*-1}=a c^{-1} d-c^{*-1}$ and hence $b d^{-1}=a c^{-1}-$ $\left(d c^{*}\right)^{-1} \in \boldsymbol{R}^{n}$ which proves $d b^{-1} \in \boldsymbol{R}^{n}$ if $b \neq 0$. If $b=c=0$, (i) and (ii) are the only conditions.

We wish to show that the Clifford matrices form a group under matrix multiplication. For this we must first make sure that a product $g_{1} g_{2}$ inherits properties (i)-(iii). For (i) we write, as a sample, $a_{1} a_{2}+b_{1} c_{2}=b_{1}\left(b_{1}^{-1} a_{1}+c_{2} a_{2}^{-1}\right) a_{2}$ which is obviously in $\Gamma_{n} \cup\{0\}$, the case where $b_{1}$ or $a_{2}=0$ being trivial. As for (ii) one shows that the pseudo-determinant $\Delta(g)=a d^{*}-b c^{*}$ is multiplicative as long as it is real. This makes $\Delta\left(g_{1} g_{2}\right)=\Delta\left(g_{1}\right) \Delta\left(g_{2}\right)=1$. In contrast, it is not obvious that $g_{1} g_{2}$ shares property (iii), and this part of the reasoning will be postponed.

The unit matrix $\left(\begin{array}{ll}1 & 0 \\ 0 & 1\end{array}\right)$ is an obvious identity. Condition (ii) together with $a b^{*}=b a^{*}$ and $c d^{*}=d c^{*}$ gives

$$
\left(\begin{array}{ll}
a & b \\
c & d
\end{array}\right)\left(\begin{array}{rr}
d^{*} & -b^{*} \\
-c^{*} & a^{*}
\end{array}\right)=\left(\begin{array}{ll}
1 & 0 \\
0 & 1
\end{array}\right)
$$


which shows that $g^{-1}=\left(\begin{array}{rr}d^{*} & -b^{*} \\ -c^{*} & a^{*}\end{array}\right)$ is a right inverse. As soon as the group property has been proved it is then also a left inverse. This can also be seen directly by showing that $d^{*} a-b^{*} c=1$.

The missing observation will become clear through a different characterization of Clifford matrices. The matrix $g=\left(\begin{array}{ll}a & b \\ c & d\end{array}\right)$ is made to act on vectors $x$ according to the rule

$$
g x=(a x+b)(c x+d)^{-1} .
$$

In order for this to make sense it is necessary to pass from $\boldsymbol{R}^{n}$ to the compactification $\overline{\boldsymbol{R}}^{n}=\boldsymbol{R}^{n} \cup\{\infty\}$. The rules for including $\infty$ are exactly the same as in the complex case. The numerator can be written as $c\left(x+c^{-1} d\right)$ and is thus visibly in $\Gamma_{n} \cup\{0\}$. The value $g x$ is never indeterminate, for $a x+b=c x+d=0$ is incompatible with $a d^{*}-b c^{*}=1$. We note that $g \infty=a c^{-1}$ and $g^{-1} \infty=-c^{-1} d$ except when $c=0$, in which case both are $\infty$.

We shall show that $g$ is a Clifford matrix if and only if (7) determines a bijective mapping $g: \overline{\boldsymbol{R}}^{n} \rightarrow \overline{\boldsymbol{R}}^{n}$. The first step is to verify that $g_{1}\left(g_{2} x\right)=\left(g_{1} g_{2}\right) x$ showing that $g_{1} g_{2}$ is bijective if $g_{1}$ and $g_{2}$ are. The second step is to note that $g$ has the factorization

$$
\left(\begin{array}{ll}
a & b \\
c & d
\end{array}\right)=\left(\begin{array}{cc}
1 & a c^{-1} \\
0 & 1
\end{array}\right)\left(\begin{array}{cc}
c^{*-1} & 0 \\
0 & c
\end{array}\right)\left(\begin{array}{cc}
0 & -1 \\
1 & 0
\end{array}\right)\left(\begin{array}{cc}
1 & c^{-1} d \\
0 & 1
\end{array}\right)
$$

The first and last factors represent parallel translations by the vectors $a c^{-1}$ and $c^{-1} d$. The second is a stretching by the factor $c^{-2}$ if $c>0$, a rotation represented by the matrix $\varrho\left(c^{\prime}\right)$ if $|c|=1$, and a product of both, in either order, if neither is the case. Finally, the third factor stands for a sense-preserving inversion $x \rightarrow-x^{-1}$. All these matrices represent simple bijective mappings of $\overline{\boldsymbol{R}}^{n}$ which are in fact generators of the sense-preserving Möbius group $M\left(\boldsymbol{R}^{n}\right)^{+}$. It follows that every Clifford matrix induces a Möbius transformation. Conversely, every sense-preserving Möbius transformation is represented by a product of factors like the ones in (8) and will therefore induce a bijection. In particular, the product of the matrices will be such that $g_{\infty}=a c^{-1}$ and $g^{-1} \infty=-c^{-1} d$ are vectors. In other words, condition (iii) is fulfilled. If $c=0$ an even simpler argument proves (iii').

All told we have sketched a proof of

Vahlen's theorem. The Clifford matrices form a group whose quotient modulo $\pm\left(\begin{array}{ll}1 & 0 \\ 0 & 1\end{array}\right)$ is isomorphic to $M\left(\boldsymbol{R}^{n}\right)^{+}$.

A Clifford matrix in dimension $n$ is also a Clifford matrix in dimension $n+1$ (and in all higher dimensions). As such it automatically extends the corresponding transformation in $M\left(\boldsymbol{R}^{n}\right)^{+}$to one in $M\left(\boldsymbol{R}^{n+1}\right)^{+}$given by the same matrix, but with $x \in \boldsymbol{R}^{n}$ replaced by $x+x_{n} e_{n} \in \boldsymbol{R}^{n+1}$. Because both mappings are sense-preserving the extension maps the upper half space $H^{n+1}$ on itself and is in fact a motion in 
hyperbolic $(n+1)$-space. This aspect is by itself a strong motivation for the use of Clifford numbers.

For later use we record the formula

$$
g x-g y=(c x+d)^{*-1}(x-y)(c y+d)^{-1}
$$

(see [Ma]), or in infinitesimal form

$$
d g x=(c x+d)^{*-1} d x(c x+d)^{-1}
$$

from which we deduce that $g^{\prime}(x)$ is the matrix $|c x+d|^{-2} \varrho\left((x c+d)^{\prime}\right)$ with the operator norm $\left|g^{\prime}(x)\right|=|c x+d|^{-2}$.

\section{General remarks on fixed points}

A vector $v \in \boldsymbol{R}^{n}$ is a fixed point of $d=\left(\begin{array}{ll}a & b \\ c & d\end{array}\right)$ if $a v+b=v(c v+d)$. Because of the lack of commutativity this equation is not trivially solvable. However, if the unknown is replaced by $c v$ the equation already looks more like an ordinary quadratic equation, and if we go one step further and introduce the unknown $\lambda=c v+d$ the equation takes the surprisingly simple form

$$
\lambda^{2}-\left(c a c^{-1}+d\right) \lambda+c c^{*-1}=0,
$$

provided that $c \neq 0$. If $v$ is a fixed point then $\lambda$ is a solution of (10). Conversely, if $\lambda$ is a solution of (10), then $v=c^{-1}(\lambda-d)$ is a fixed point provided that $c^{-1} \lambda$ is a vector.

In what follows we shall use the same letter for a matrix $g$ and the transformation it induces. The fact that $g$ and $-g$ induce the same mapping is usually irrelevant and can be ignored. If $h$ is another Clifford matrix, then $g$ and $h g h^{-1}$ are conjugate. If $g$ has the fixed point $v$, then $h g h^{-1}$ has the fixed point $h v$. For this reason the whole fixed point problem is the same for conjugate matrices, and in fact for the whole conjugacy class.

The point $\infty$ is a fixed point of $g$ if and only if $c=0$, and 0 is a fixed point if $b=0$. If both are fixed points $g$ will be of the form $\left(\begin{array}{cc}\lambda & 0 \\ 0 & \lambda^{*-1}\end{array}\right)$. A matrix $g$ is diagonalizable if it is conjugate to a matrix of this form. Suppose that $h g h^{-1}=\left(\begin{array}{cc}\lambda & 0 \\ 0 & \lambda^{*-1}\end{array}\right)$. Then $g$ has the fixed points $u=h^{-1} 0$ and $v=h^{-1} \infty$. Here $h$ is not unique, but we make the specific choice

$$
h=h_{u, v}=\left(\begin{array}{cc}
1 & -u \\
(u-v)^{-1} & -(u-v)^{-1} v
\end{array}\right) .
$$


An easy computation, which makes use of $u$ and $v$ being fixed points, leads to

$$
h_{u, v} g h_{u, v}^{-1}=\left(\begin{array}{cc}
(u-v)(c v+d)(u-v)^{-1} & 0 \\
0 & c u+d
\end{array}\right) .
$$

Because $a d^{*}-b c^{*}=1$ for all Clifford matrices it follows from (12) that $c u+d$ and $c v+d$ are simultaneously real, and that $|c u+d|=1$ implies $|c v+d|=1$. This will play an important role.

The isometric sphere $S(g)$ is the set of points where $\left|g^{\prime}(x)\right|=1$, i.e., $|c x+d|=1$. It is an $S^{n-1}$ with radius $|c|^{-1}$ and center $g^{-1} \infty=-c^{-1} d$. Similarly, $S\left(g^{-1}\right)$ has the same radius and the center $g \infty=a c^{-1}$. As in the complex case, $g$ maps $S(g)$ isometrically on $S\left(g^{-1}\right)$. A fixed point which lies on one of the isometric spheres will necessarily lie on their intersection.

Conjugation by parallel translation has a particularly simple effect and can be used to achieve an interesting and helpful symmetry. If $\beta$ is a vector one finds

$$
\left(\begin{array}{ll}
1 & \beta \\
0 & 1
\end{array}\right) g\left(\begin{array}{rr}
1 & -\beta \\
0 & 1
\end{array}\right)=\left(\begin{array}{cc}
a+\beta c & * \\
c & -c \beta+d
\end{array}\right)
$$

where the element marked with a star is unimportant. The important observation is that $c$ has not changed, and neither has the quantity $g \infty-g^{-1} \infty=a c^{-1}+c^{-1} d$. It is convenient to introduce the notation $\sigma=(1 / 2)\left(a c^{-1}+c^{-1} d\right)$. With this notation (10) is replaced by

$$
\lambda^{2}-2(c \sigma) \lambda+c c^{*-1}=0
$$

If we choose $\beta=(1 / 2)\left(c^{-1} d-a c^{-1}\right)$ in (13) the matrix on the right will be of the form

$$
g_{0}=\left(\begin{array}{cc}
\sigma c & \sigma c \sigma-c^{*-1} \\
c & c \sigma
\end{array}\right)
$$

where the element on the upper right has been calculated from $\Delta\left(g_{0}\right)=1$. We shall say that $g_{0}$ is normalized, and we have shown that every Clifford matrix with $c \neq 0$ is conjugate to a normalized matrix. If $g_{0}$ has a fixed point $v_{0}$, then the original has the fixed point $v_{0}+(1 / 2)\left(a c^{-1}-c^{-1} d\right)$. As far as fixed points are concerned it is therefore sufficient to consider normalized matrices.

If $g$ is normalized an easy calculation shows that $v$ is a fixed point if and only if it satisfies the condition

$$
c(v+\sigma) c^{*}(v-\sigma)=-1
$$

The symmetry is complete if $c=c^{*}$. In that case $v$ and $-v$ are simultaneously fixed points. Condition (16) replaces (14), but in some connections (14) is easier to use. 


\section{The hyperbolic case}

A Clifford matrix, and the corresponding Möbius transformation, is called hyperbolic if it is conjugate to a matrix $\left(\begin{array}{cc}\lambda & 0 \\ 0 & \lambda^{-1}\end{array}\right)$ where $\lambda$ is real and different from \pm 1 . Except for changing the sign or interchanging $\lambda$ and $\lambda^{-1}$, it is the only diagonal matrix in its conjugacy class.

If $g$ has the fixed points $u$ and $v$ it follows from (12) that $c u+d$ and $c v+d$ are, except for sign, equal to $\lambda$ and $\lambda^{-1}$ in either order.

Theorem A. The matrix $g=\left(\begin{array}{ll}a & b \\ c & d\end{array}\right)$ with $c \neq 0$ is hyperbolic if and only if $c \sigma$ is real and $(c \sigma)^{2}>1$. When this is so it is also true that $a+d^{*}$ is real and of $a b-$ solute value $>2$.

Proof. If $g$ is hyperbolic with fixed point $v$ we may assume that $c v+d=\lambda>0$. It follows that $c^{-1}=\lambda^{-1}\left(v+c^{-1} d\right)$ is a vector, and hence that $c=c^{*}$. From (10) we conclude that $2 c \sigma=c a c^{-1}+d=\lambda+\lambda^{-1}$ which is real and greater than 2 , so that $(c \sigma)^{2}>1$. Furthermore, $a c^{-1}=c^{*-1} a^{*}$ so that $c a c^{-1}+d=a^{*}+d=a+d^{*}>2$. Conversely, $c \sigma$ real implies that $c$ is a vector and $c=c^{*}$. Together with $(c \sigma)^{2}>1$ it becomes clear that (14) has two real roots $\lambda, \lambda^{-1}$ which correspond to vectors $u, v$, and (12) shows that $g$ is hyperbolic.

From the explicit solutions $\lambda=c \sigma \pm\left((c \sigma)^{2}-1\right)^{1 / 2}$

$$
u, v=\frac{1}{2}\left(a c^{-1}-c^{-1} d\right) \pm \frac{1}{2} c^{-1}\left(\left(a+d^{*}\right)^{2}-4\right)^{1 / 2},
$$

in close analogy with the complex case. If $g$ is normalized the first term is missing.

The inequality $|c \sigma|>1$ expresses the fact that the isometric spheres do not intersect. The sphere with $u$ and $v$ as the end points of a diameter intersects the isometric spheres at right angles.

Remark. If $c=0$ but $b \neq 0$ the theorem is applicable to $\left(\begin{array}{rr}d & 0 \\ -b & a\end{array}\right)$ which is conjugate to $g$. If $b=c=0$ we know from the start that $g$ is hyperbolic, with fixed points 0 and $\infty$, if and only if $a$ and $b$ are of the form $\lambda, \lambda^{-1}$ with $\lambda$ real and $\neq \pm 1$.

\section{The elliptic case}

It is natural to say that a Clifford matrix is elliptic if it is conjugate to one of the form $\left(\begin{array}{ll}\lambda & 0 \\ 0 & \lambda\end{array}\right),|\lambda|=1, \lambda \neq \pm 1$. In this form the definition is ambiguous, unless one specifies in what dimension the conjugation has to take place. Our choice is to say that a Clifford matrix $g$ in dimension $n$ is elliptic in $\boldsymbol{R}^{n}$ if there exists a matrix 
$h$, again in dimension $n$, such that $h g h^{-1}$ is of the required form. It is of course permissible to consider the action of $g$ in $\boldsymbol{R}^{n+1}$, and it can happen that $g$ is elliptic in $\boldsymbol{R}^{n+1}$, but not in $\boldsymbol{R}^{n}$.

If $g$ is elliptic in $\boldsymbol{R}^{n}$ the discussion in Section 3 has already shown that $g$ has at least two fixed points $u$ and $v$. If $c \neq 0$ they are both finite, and we conclude from (12) that $|c u+d|=|c v+d|=1$. In other words, all fixed points must lie on the intersection of the isometric spheres $S^{n-1}(g)$ and $S^{n-1}\left(g^{-1}\right)$. Because the intersection must contain at least two points, all hyperbolic matrices satisfy the basic condition $|\sigma c|<1$.

We assume again that $g$ is normalized, $a c^{-1}=c^{-1} d=\sigma$. The case $\sigma=0$ is exceptional and will be treated separately. When $\sigma \neq 0$ the intersection of the isometric spheres is an $S^{n-2}$, situated in the hyperplane through the origin perpendicular to $\sigma$, with center 0 and radius $r$ given by $r^{2}=|c|^{-2}-|\sigma|^{2}$. In the sequel, $S^{n-2}(r)$ will refer to this particular $(n-2)$-sphere; it reduces to two points when $n=2$.

The axis of an $a \in \Gamma_{n}$ consists of all $x \in \boldsymbol{R}^{n} \backslash\{0\}$ with $x=a x a^{-1}$, i.e., the eigenvectors of $\varrho(a)$ for the eigenvalue 1 . The axis will be denoted by $V(a)$. Because $\varrho(a)$ is sense-preserving, the codimension of $V(a)$ is even. Therefore $\operatorname{dim} V(a) \leqq n-2$ except when $a$ is real, in which case $V(a)=\boldsymbol{R}^{n} \backslash\{0\}$. It is a purely algebraic task to determine $V(a)$ explicitly when $a$ is given, for instance as a product of vectors in $\boldsymbol{R}^{n}$.

Theorem B. Assume that $g$ is normalized and $\sigma c \neq 0$. Let $\varphi$ denote the angle between $\sigma$ and its orthogonal projection on $V(\sigma c)$. Then $g$ is elliptic in $\boldsymbol{R}^{n}$ if and only if $\operatorname{dim} V(\sigma c) \geqq 2$ and $|\sigma c|<\cos \varphi$.

The set of fixed points is the intersection of $S^{n-2}(r)$ and $-\sigma+V(\sigma c)$. If $\operatorname{dim} V(\sigma c)=k$ it is a sphere $S^{k-2} \subset S^{n-2}(r)$ with radius $r \cos \varphi$. For $k=2$ it reduces to two points.

Proof. We know that $v$ is a fixed point if and only if it satisfies (16). If $g$ is elliptic we also know that $v$ lies on $S^{n-1}(r)$, which means that $v$ is perpendicular to $\sigma$ and $|v+\sigma|=|v-\sigma|=|c|^{-1}$. Subject to this condition (16) can be replaced by an equivalent property with a more obvious interpretation.

The fact that $v$ is perpendicular to $\sigma$ is expressed by $\sigma \bar{v}=-v \bar{\sigma}$, or $\bar{v} \bar{\sigma}^{-1}=-\sigma^{-1} v$. This leads to

$$
|c|^{-2}(v-\sigma)^{-1}=\left(\bar{v} \bar{\sigma}^{-1}-1\right) \bar{\sigma}=-\left(\sigma^{-1} v+1\right) \bar{\sigma}=-\sigma^{-1}(v+\sigma) \bar{\sigma},
$$

and it follows that (16) can be replaced by

$$
\sigma c(v+\sigma)(\sigma c)^{\prime-1}=v+\sigma .
$$

In other words, $v+\sigma$ lies on the axis of $V(\sigma c)$, as asserted in the second part of the theorem. 
If $g$ is elliptic there are at least two fixed points. It follows that $-\sigma+V(\sigma c)$ must meet $S^{n-2}(r)$ in more than one point. This is impossible unless $V(\sigma c)$ is of dimension $k>1$. It is readily seen that the parallel to $V(\sigma c)$ through $-\sigma$ intersects the ball $B^{n-1}(r)$ if and only if $|\sigma| \tan \varphi<r$, which is the same as $|\sigma c|<\cos \varphi$. Under this condition it will intersect $S^{n-2}(r)$ in a $(k-2)$-sphere of radius $r \cos \varphi$. This is the set of fixed points.

For the converse, all one needs is to observe that $|\sigma c|<\cos \varphi$ and $k>1$ guarantees the existence of two points $u$ and $v$ which lie on $S^{n-2}(r)$ and satisfy (17). It can then be read off from (12) that $g$ is conjugate to a matrix $\left(\begin{array}{ll}\lambda & 0 \\ 0 & \lambda^{\prime}\end{array}\right)$ with $|\lambda|=1$ and is thus elliptic.

The result is particularly simple if $\sigma$ belongs to the axis $V(\sigma c)$, in which case $\varphi=0$.

Corollary. The vector $\sigma \in V(\sigma c)$ if and only if $c \sigma=(\sigma c)^{\prime}$. When this is so, the condition $|\sigma c|<1$ is necessary and sufficient for $g$ to be elliptic in $\boldsymbol{R}^{n}$ if $n$ is even, in $\boldsymbol{R}^{n+1}$ if $n$ is odd.

We are still assuming $\sigma \neq 0$. The condition $\sigma \in V(\sigma c)$ reads $\sigma c \sigma=\sigma(\sigma c)^{\prime}$ which is the same as $c \sigma=(\sigma c)^{\prime}$. If $n$ is even, so is $k$. Since $\sigma$ is in $V(\sigma c)$ it follows that $k \geqq 2$ and, by the theorem, $g$ is elliptic in $\boldsymbol{R}^{n}$. If $n$ is odd $n+1$ is even, and $g$ is elliptic in $\boldsymbol{R}^{n+1}$. More explicitly, $\sigma$ and $e_{n}$ are linearly independent vectors in $V(\sigma c)$ so that $g$ is always elliptic in $\boldsymbol{R}^{n+1}$ regardless of the parity.

For $n=2$, the classical complex case, $\sigma c=(a+d) / 2$ and the corollary reduces to the familiar fact that $g$ is elliptic if and only if the trace is real and of absolute value $<2$.

It remains to consider the case $\sigma c=0$. If $c=0$ and $b \neq 0$ the theorem applies to $\left(\begin{array}{rr}d & 0 \\ -b & a\end{array}\right)$ which is conjugate to $g$ by means of $\left(\begin{array}{rr}0 & 1 \\ -1 & 0\end{array}\right)$. If $b=c=0, g$ is elliptic if $|a|=1$, and $v$ is a fixed point if it belongs to $V(a)$.

If $\sigma=0$ and $g$ is normalized the isometric spheres coincide with each other and with $S^{n-1}\left(|c|^{-1}\right)$. From $g=\left(\begin{array}{cc}0 & -c^{*-1} \\ c & 0\end{array}\right)$ it is seen that $v$ is a fixed point if and only if $|v|=|c|^{-1}$ and $\varrho(c) v=-\bar{v}$. The mapping $x \rightarrow-\bar{x}$ is sense-reversing with matrix $J=\left(\begin{array}{rl}-1 & 0 \\ 0 & 1_{n-1}\end{array}\right)$. The fixed points will be eigenvectors of $\varrho(c) J$ for the eigenvalue 1. If $n$ is even 1 is an eigenvalue, and $g$ is elliptic in $\boldsymbol{R}^{n}$. If $n$ is odd $g$ is elliptic in $\boldsymbol{R}^{n+1}$. 


\section{The parabolic case}

A Möbius transformation is said to be parabolic if it has one and only one fixed point in $\overline{\boldsymbol{R}}^{n}$. We shall use an alternate definition which is more in harmony with our preference for normalized matrices. The equivalence of the definitions is stated as a lemma. The proof follows a suggestion by B. Maskit.

Lemma. A Clifford matrix has a single fixed point if and only if it is conjugate to a normalized matrix with fixed point 0.

Proof. A normalized matrix with fixed point 0 is of the form $\left(\begin{array}{cc}\sigma c & 0 \\ c & c \sigma\end{array}\right)$ with $\sigma \in \boldsymbol{R}^{n}$ and $\sigma c(c \sigma)^{*}=\sigma c \sigma c^{*}=1$. This condition can also be written either as $(\sigma c)^{2}=$ $c c^{*-1}$ or as $\sigma c=(c \sigma)^{\prime}$ together with $|\sigma c|=1$. We show first that there are no other fixed points.

A fixed point $v$ would satisfy $\sigma c v=v c v+v c \sigma$, from which $|\sigma|=|v+\sigma|$. The same relation written as $\sigma c v-v c v=v c \sigma$ implies $|v-\sigma|=|\sigma|$. The two conditions are incompatible unless $v=0$. Hence 0 is the only fixed point.

Suppose next that $g$ has a single fixed point. As far as the lemma is concerned we may assume that the fixed point is at $\infty$, in which case $g=\left(\begin{array}{cc}a & b \\ 0 & a^{*-1}\end{array}\right)$. In matrix language a fixed point $v$ would satisfy $\left(I-|a|^{2} \varrho(a)\right) v=b a^{*}$. If $|a|<1$ the matrix $I-|a|^{2} \varrho(a)$ has an inverse and the equation can be solved for $v$. If $|a|>1$ the same reasoning applies to $g^{-1}$. It follows that $g=\left(\begin{array}{cc}a & u a^{\prime} \\ 0 & a^{\prime}\end{array}\right)$ with $|a|=1, g \infty=u \in \boldsymbol{R}^{n}$.

The axis $V(a)$ is the null-space of $I-\varrho(a)$. The null-space and the range of $I-\varrho(a)$ are orthogonal complements. It is therefore possible to write $u=u_{p}-$ $\left(\beta-a \beta a^{\prime-1}\right)$ where $u_{p}$ is the orthogonal projection of $u$ on $V(a)$ and $\beta \in \boldsymbol{R}^{n}$. Translation by $\beta$ transforms $g$ into

$$
\left(\begin{array}{ll}
1 & \beta \\
0 & 1
\end{array}\right) g\left(\begin{array}{rr}
1 & -\beta \\
0 & 1
\end{array}\right)=\left(\begin{array}{cc}
a & u a^{\prime}-a \beta+\beta a^{\prime} \\
0 & a^{\prime}
\end{array}\right)=\left(\begin{array}{cc}
a & u_{p} a^{\prime} \\
0 & a^{\prime}
\end{array}\right)
$$

Here $u_{p} \neq 0$ since otherwise $g$ would have the fixed point $-\beta$. Conjugation by $\left(\begin{array}{rr}0 & 1 \\ -1 & 0\end{array}\right)$ changes the last matrix to $\left(\begin{array}{cc}a^{\prime} & 0 \\ -u_{p} a^{\prime} & a\end{array}\right)$. Because $u_{p} a^{\prime}=a u_{p}$ this matrix is normalized with fixed point 0 and $\sigma=-u_{p}^{-1}$. We have shown that $g$ is conjugate to a matrix of the required type.

In the following theorem $\varphi$ will again denote the angle between $\sigma$ and its projection $\sigma_{p}$ on $V(\sigma c)$. The proof is very similar to that of Theorem B.

Theorem C. The matrix $g=\left(\begin{array}{ll}a & b \\ c & d\end{array}\right)$ with $\sigma c \neq 0$ is parabolic if and only if $V(\sigma c) \neq 0$ and $|\sigma c|=\cos \varphi$. The fixed point is $-\sigma+|\sigma c|^{-2} \sigma_{p}$. 
Proof. It is no restriction to assume that $g$ is normalized. Suppose that $v$ is the only fixed point of $g$. Conjugation by $h=\left(\begin{array}{rr}0 & 1 \\ -1 & v\end{array}\right)$ throws the fixed point to $\infty$ and results in

$$
h g h^{-1}=\left(\begin{array}{cc}
c v+d & -c \\
0 & a-v c
\end{array}\right)=\left(\begin{array}{cc}
c(v+\sigma) & -c \\
0 & (\sigma-v) c
\end{array}\right) .
$$

As in the proof of the Lemma this implies $|v+\sigma|=|v-\sigma|=|c|^{-1}$ so that $v$ lies on the intersection $S^{n-2}(r)$ of the isometric spheres. In addition, $c(v+\sigma) c^{*}(\sigma-v)=1$, which is nothing else than (16).

Exactly as in the proof of Theorem B, a point $v \in S^{n-2}(r)$ is a fixed point if and only if $v+\sigma \in V(\sigma c)$. Therefore, if $g$ is parabolic $S^{n-2}(r)$ and $-\sigma+V(\sigma c)$ meet in a single point. Since $v \neq-\sigma$ it follows that $V(\sigma c) \neq 0$. It is also geometrically evident that $r=|\sigma| \tan \varphi$, or $|\sigma c|=\cos \varphi$. Conversely, if this is so there is a single common point $v$ which must be a fixed point. It follows by (12) that any other fixed point $u$ would satisfy $|c u+d|=1$ and hence also lie on $S^{n-2}(r)$ and on $-\sigma+V(\sigma c)$. The contradiction proves that $g$ is parabolic.

\section{An illustration}

Sections 5-7 provide a recipe for recognizing hyperbolic, elliptic, and parabolic transformations. It is nevertheless useful to consider an explicit example which shows how the nature of a Möbius transformation changes with a varying parameter.

Because $\sigma$ is a vector it is of course always possible to choose coordinates so that $\sigma$ is real and $\geqq 0$. From our point of view it is essential that the change of coordinates be given in terms of the coefficients. We assume $\sigma \neq 0$. A rotation by $\varrho(\alpha)$, $|\alpha|=1$, replaces $g=\left(\begin{array}{ll}a & b \\ c & d\end{array}\right)$ by $\left(\begin{array}{cc}\alpha a \bar{\alpha} & \alpha b \alpha^{*} \\ \alpha^{\prime} c \bar{\alpha} & \alpha^{\prime} d \alpha^{*}\end{array}\right)$ and hence $\sigma$ by $\alpha \sigma \alpha^{*}$. This is positive if $\alpha \sigma \alpha^{*}=|\sigma|$, or $\bar{\alpha} \alpha^{\prime}=\sigma /|\sigma|$. If $\alpha$ is a vector this becomes $\alpha^{2}=\bar{\sigma} /|\sigma|$. Therefore, if the square root exists and is a vector, the choice $\alpha=(\bar{\sigma} /|\sigma|)^{1 / 2}$ leads to the desired result.

It is a basic result that every vector $s$ has a square root which is itself a vector. To see this we denote the real part of $s$ by $s_{0}$ and observe that $\left|s-s_{0}\right|^{2}=-\left(s-s_{0}\right)^{2}$. If $s \neq s_{0}$ there are two square roots given by the formula

$$
\sqrt{s}= \pm\left(\left(\frac{|s|+s_{0}}{2}\right)^{1 / 2}+\frac{s-s_{0}}{\left|s-s_{0}\right|}\left(\frac{|s|-s_{0}}{2}\right)^{1 / 2}\right) .
$$

The verification makes use of $|s|^{2}=s_{0}^{2}+\left|s-s_{0}\right|^{2}$. A positive number has its usual two square roots, and a negative number has infinitely many if $n>2$, namely $|s|\left(t_{1} e_{1}+\ldots+t_{n-1} e_{n-1}\right)$ with $|t|=1$. We have shown that there is no loss in generality in choosing $\sigma$ to be real. 
Our example will be a normalized Clifford matrix with $n=3, \sigma>0$, and $c=$ $\left(1+e_{1}\right) e_{2}$. Note that $|c|=\sqrt{2}$. We shall also need $c c^{*}=-2 e_{1}, c e_{1} c^{*}=-2, c e_{2} c^{*}=$ $-2 e_{2}, c e_{3} c^{*}=2 e_{3}$ from which it follows that

$$
\varrho(\sigma c)=\varrho(c)=\left(\begin{array}{rrrr}
0 & -1 & 0 & 0 \\
-1 & 0 & 0 & 0 \\
0 & 0 & -1 & 0 \\
0 & 0 & 0 & 1
\end{array}\right) .
$$

The axis $V(\sigma c)$ in $\boldsymbol{R}^{3}$ is spanned by $1+e_{1}$ and the axis in $\boldsymbol{R}^{4}$ by $1+e_{1}$ and $e_{3}$. In either case $\varphi=45^{\circ}$.

We shall determine all fixed points in $\boldsymbol{R}^{4}$, and for this purpose we make use of (16) which we prefer to write as

$$
c(v+\sigma) c^{*}=-(v-\sigma)^{-1}=-\frac{\bar{v}-\sigma}{|v-\sigma|^{2}} .
$$

With $v=v_{0}+v_{1} e_{1}+v_{2} e_{2}+v_{3} e_{3}$ (18) becomes

$$
-v_{1}-\left(v_{0}+\sigma\right) e_{1}-v_{2} e_{2}+v_{3} e_{3}=\frac{1}{2|v-\sigma|^{2}}\left(-\left(v_{0}-\sigma\right)+v_{1} e_{1}+v_{2} e_{2}+v_{3} e_{3}\right)
$$

and thus

$$
v_{1}=\frac{v_{0}-\sigma}{2|v-\sigma|^{2}}, \quad v_{0}+\sigma=-\frac{v_{1}}{2|v-\sigma|^{2}}, \quad v_{2}=-\frac{v_{2}}{2|v-\sigma|^{2}}, \quad v_{3}=\frac{v_{3}}{2|v-\sigma|^{2}} .
$$

It is clear at once that $v_{2}=0$. The first two equations give $v_{0}^{2}+v_{1}^{2}=\sigma^{2}$. If we are looking only for fixed points in $\boldsymbol{R}^{3}, v_{3}=0$ and $|v-\sigma|^{2}=2 \sigma\left(\sigma-v_{0}\right)$. This makes $v_{1}=-1 / 4 \sigma$ and $v_{0}= \pm(1 / 4 \sigma)\left(16 \sigma^{4}-1\right)^{1 / 2}$ provided that $\sigma \geqq 1 / 2$. For $\sigma>1 / 2$ the transformation is loxodromic with fixed points $(1 / 4 \sigma)\left[ \pm\left(16 \sigma^{4}-1\right)^{1 / 2}-e_{1}\right]$. If $\sigma=1 / 2$ it is parabolic with the fixed point $-e_{1} / 2$. This agrees with $|\sigma c|>\cos \varphi$ in the loxodromic case and $|\sigma c|=\cos \varphi$ in the parabolic case. The parabolic example is particularly important because it shows that the isometric spheres of a parabolic transformation need not be tangent to each other.

If we look for fixed points in $\boldsymbol{R}^{4}$ we have to include the case $v_{3} \neq 0$ which leads to $|v-\sigma|^{2}=1 / 2, v_{0}=0, v_{1}=-\sigma$, and $v_{3}^{2}=1 / 2-2 \sigma^{2}$. For any $0 \leqq \sigma<1 / 2$ it follows that $g$ is hyperbolic in $\boldsymbol{R}^{4}$ with fixed points $-\sigma \pm\left(\left(1-4 \sigma^{2}\right) / 2\right)^{1 / 2} e_{3}$.

Acknowledgements. I am indebted to Dennis Hejhal who found the paper of Vahlen, and to Pertti Lounesto who made me acquainted with the more recent work on Clifford algebras. I am also grateful to Troels Jørgensen for many discussions, and very particularly to Bernie Maskit who helped me clear up some misconceptions about parabolic transformations. 


\title{
References
}

[Ag] Agard, S.: A geometric proof of Mostow's rigidity theorem for groups of divergence type. - Acta Math. 151, 1983, 231-252.

[Ah 1] AhLFORs, L.: Möbius transformations and Clifford numbers. - Differential geometry and complex analysis, H. E. Rauch memorial volume. Springer-Verlag, Berlin, 1985.

[Ah 2] Ahlfors, L.: Old and new in Möbius groups. - Ann. Acad. Sci. Fenn. Ser. A. I. Math. 9, 1984, 93-105.

[Fi] Fillmore, J. P.: The fifteen-parameter conformal group. - Internat. J. Theoret. Phys. 16, 1977, 937-963.

[Ha] Haffeli, H. G.: Hyperkomplexe differentiale. - Comment. Math. Helv. 20, 1947, 382420.

[Lo-La] Lounesto, P., and E. Latvamaa: Conformal transformations and Clifford algebras. Proc. Amer. Math. Soc. 79, 1980, 533-538.

[Ma] MaAss, H.: Automorphe Funktionen von mehreren Veränderlichen und Dirichletsche Reihen. - Abh. Math. Sem. Univ. Hamburg 16:3/4, 1949, 72-100.

[Po] Porteous, I. R. : Topological geometry, 2nd ed. - Cambridge University Press, Cambridge, 1981.

[Va] VAhLEN, K. Th.: Über Bewegungen und complexe Zahlen. - Math. Ann. 55, 1902, 585-593.

[Wi] Wielenberg, N. J.: Discrete Moebius groups: Fundamental polyhedra and convergence. Amer. J. Math. 99, 1977, 861-877.

\author{
Harvard University \\ Department of Mathematics \\ Cambridge, Mass. 02138 \\ USA
}

Received 7 May 1984 